\title{
Lasing in Dye-Doped Photonic Liquid Crystal Devices
}

\author{
Yuhua Huang \\ Ying Zhou \\ Shin-Tson Wu \\ College of Optics and Photonics, University of Central Florida, \\ Orlando, Florida, USA
}

We have investigated the effects of pumping laser's polarization and incident angles, dye concentration, cell gap, and lasing wavelength on the lasing efficiency of the dye doped cholesteric liquid crystal (CLC) devices. These factors impact the lasing efficiency due to the existence of the photon bleaching and quench in the dye molecules, the competition of the optical gain and loss in the media, and the helical layers dependent amplification provided by the distributed feedback. The maximum lasing efficiency ( 0.5\%) was obtained at $\lambda \sim 608 \mathrm{~nm}$ from a $10 \mu \mathrm{m}$ CLC sample doped with $1 \mathrm{wt} \%$ DCM when the pump laser beam is left-handed circularly polarized and the incident angle is normal to the sample.

Keywords: cholesteric liquid crystal; laser; lasing efficiency; photonic liquid crystal

\section{INTRODUCTION}

Photonic crystals with periodic dielectric structures have attracted great interest from both scientific and technological viewpoints [1-6]. Many new physical phenomena including suppression of spontaneous emission, absence of zero fluctuation, energy transfer, bound state of photonic, optical switching and bistability can occur in the structure possessing gaps in the photonic band gap structure. These significant properties provide the possibility for these artificial photonic crystals to improve the performance of an electro-optic device. Thus, the existence of the spectral gap in such photonic crystals opens up a variety of possible potential applications such as thresholdless semiconductor lasers, efficient optical filters, air-core fiber, etc. [7-9]. However, the fabrication process of photonic crystal is usually very complicated

Address correspondence to Shin-Tson Wu, College of Optics and Photonics, University of Central Florida, Orlando, Florida 32816, USA. E-mail: swu@mail.ucf.edu 
and the light behavior in photonic band gap structure is very sensitive to geometry and media parameters. Therefore, it is very difficult to design and fabricate photonic band gap structure in photonic crystals with desired characteristics.

Cholesteric liquid crystal (CLC) is a medium with self-organized periodic helical structure [10]. Since LC is a highly birefringent medium, the periodic helical structure gives a periodic modulation of the refractive index and consequently offers a one-dimensional (1D) photonic band gap with the central wavelength at $\lambda_{o}=\langle n\rangle p$, where $p$ is the helical pitch and $\langle n\rangle$ the average refractive index. A CLC cell is typically prepared by doping some chiral agents into a nematic LC mixture. The fabrication process is quite simple and the electromagnetic characteristics of CLC photonic band gap can be easily controlled by adjusting the LC or chiral parameters, or by adjusting external factors such as temperature, pressure, or electric field [11-14]. Therefore, CLC is a promising $1 \mathrm{D}$ photonic crystal candidate. Recently, they have been extensively studied for mirrorless feedback laser applications [14-16].

In this paper, we investigate the effect of pumping laser's polarization and incident angles, dye concentration, cell gap, and lasing wavelengths on the lasing efficiency of the dye-doped CLC with right-handed helixes. Results show that the lasing efficiency strongly depends on dye concentration, cell gap, and lasing wavelengths. We obtained the optimal lasing efficiency $(\sim 0.5 \%)$ at $\lambda \sim 608 \mathrm{~nm}$ from a $10 \mu \mathrm{m}$ cell with $1 \mathrm{wt} \%$ dye-doped CLC mixture.

\section{SAMPLE PREPARATION AND EXPERIMENTAL SETUP}

The fabrication process of dye-doped CLC samples is described as follows: nematic LC mixture BL006, chiral MLC-6248 (both are from Merck), and laser dye 4-(dicyanomethylene)-2-methyl-6-(4-dimethylaminostryl)-4H-pyran (DCM, Exciton) were used in this study. To shift the lasing wavelength, we prepared several different CLC hosts with chiral concentration ranging from 25.7 to $27.7 \mathrm{wt} \%$ and then added $1 \mathrm{wt} \%$ DCM in each CLC mixture. To study the dye concentration effect on the lasing efficiency, we mixed $0.5,0.75,1,1.5$, and $2 \mathrm{wt} \%$ DCM into the CLC host while keeping the chiral MLC-6248 at $26.8 \mathrm{wt} \%$. Three cells with $8-\mu \mathrm{m}, 10-\mu \mathrm{m}$ and $15-\mu \mathrm{m}$ gaps were used for studying the cell gap effect.

Figure 1 shows the experimental setup. A second harmonic Q-switched Nd:YAG pulsed laser (from Continuum) at $\lambda=532 \mathrm{~nm}$ with vertical linear polarization was used to excite the dye-doped CLC sample. The pulse width is $6 \mathrm{~ns}$ and repetition rate is $1 \mathrm{~Hz}$. The 


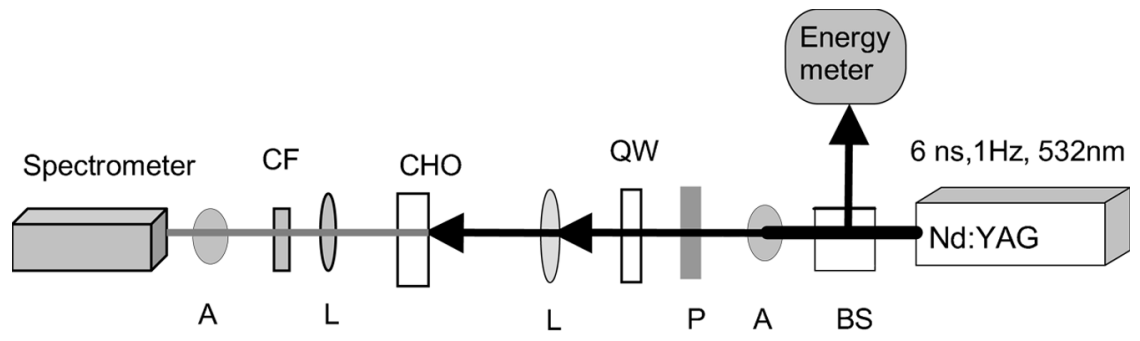

FIGURE 1 Experimental setup. BS: beam splitter, QW: quarter-wave plate, A: attenuator, CF: color filter, CHO: dye-doped cholesteric LC and L: lens.

reason that we chose $1 \mathrm{~Hz}$ repetition rate is to avoid sample heating and degradation. A beam splitter was used to divide the incoming laser beam into two beams. One was detected by a laser energy meter (Ophir) for monitoring the pumping pulse energy and the other was used as the excitation beam. A linear polarizer and a quarter-wave plate were used to convert the linear polarization (LP) into left-handed (LH) circular polarization (CP) to avoid the reflection by the photonic band gap. A lens with $150 \mathrm{~mm}$ focal length focused the incident beam to a small spot with $\sim 160 \mu \mathrm{m}$ diameter at the sample. The output laser emission in the forward direction of the sample was collected by a lens to a fiber-optics based universal serial bus (USB) spectrometer (resolution $=0.04 \mathrm{~nm}$; USB HR2000, Ocean Optics). Since the output laser emission from the sample is always normal to the sample surface, in order to avoid any problematic optics adjustment during the incident angle change, we mounted the sample, lens, head of the fiber-based spectrometer and other necessary optical components including color filters and attenuators on a rail. In this case, the incident angle could be easily changed by rotating the rail without disturbing the adjustment of optics.

\section{EXPERIMENTAL RESULTS AND DISCUSSION}

First, we characterized the transmittance and lasing emission spectra of each dye-doped CLC sample. Here we took the dye-doped CLC with $25.5 \mathrm{wt} \%$ MLC-6248 as an example to show the relation between the transmittance (using an unpolarized light) and lasing emission spectra. Results are plotted in Figure 2. This sample exhibits a reflection band from $550 \mathrm{~nm}$ to $635 \mathrm{~nm}$ and a single lasing peak near the long wavelength edge of the reflection band when the sample is excited by the pulsed laser. The reason is that the circularly polarized optical wave with the same handedness as the helical structure is evanescent 


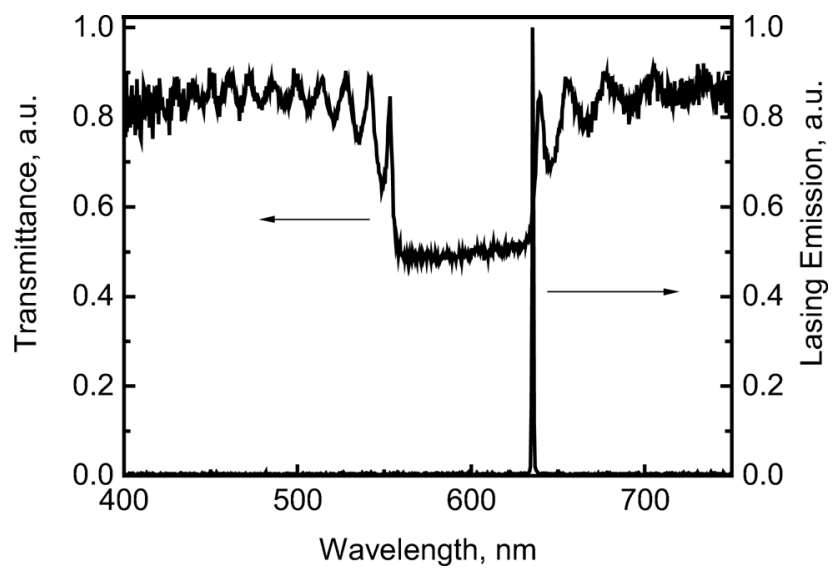

FIGURE 2 Transmission spectra of the dye-doped CLC cell and the laser emission line.

within the reflection band, corresponding to a vanishing density of state and the opposite polarized light is uniformly transmitted. Since the optical emission is proportional to the density of state, it is suppressed within the reflection band, but enhanced at the band edge. For this reason, lasing in dye-doped CLC cells always occurs at either edge of the stop band rather than at its center.

Since the CLC sample is right-handed $(\mathrm{RH})$, it reflects the $\mathrm{RH} \mathrm{CP}$ light within its reflection band but does not affect the propagation of the LH CP light. First, we studied the effect of the pumping light polarization on the CLC lasing emission at various incident angles.

Figure 3(a) shows the lasing behavior of the CLC sample under the green laser excitation at vertical linear (squares), $\mathrm{RH}$ (triangles), and LH (circles) circular polarization at normal incidence $\left(\theta=0^{\circ}\right)$. As shown in Figure 3(a), at normal incidence the lasing efficiency is almost the same for all the three different polarization states when compared at the same pumping energy. This indicates that the laser emission is basically polarization independent.

In our experiment, we found that this polarization-independent lasing phenomenon extends to $|\theta| \leq 18^{\circ}$. As the incident angle exceeds $\pm 18^{\circ}$, the lasing properties strongly depend on the polarization state of the excitation laser. Here let us take the results at $\theta=27^{\circ}$ (in air) as an example. As illustrated in Figure 3(b), the LH CP light has the lowest pumping threshold, followed by the LP light and then the RH CP light. Accordingly, at a given green laser energy, the LH circularly polarized light has the highest efficiency of red laser emission 

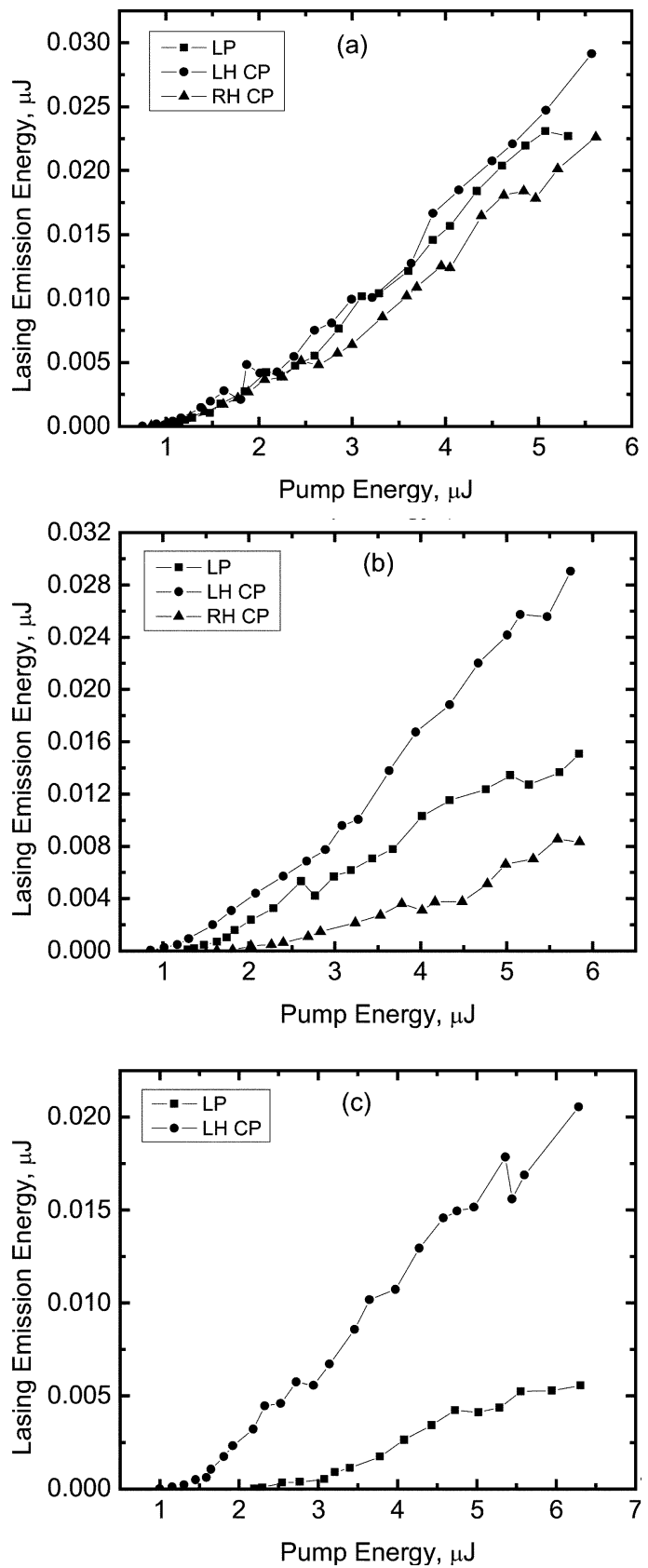

FIGURE 3 Laser emission at different incident angles: (a) $0^{\circ}$, (b) $27^{\circ}$ and (c) $31^{\circ}$. 
and the RH CP light has the lowest efficiency. Further increasing the incident angle by rotating the rail enlarges the difference between the LH CP, LP, and RH CP pumping lights. Especially when the incident angle is above $31^{\circ}$, laser action stops for the RH CP laser pumping. The lasing intensity excited by the LH CP light is much higher than that by the LP light, as Figure 3(c) depicts.

To understand the above phenomenon, we measured the transmittance spectrum of a CLC cell with $26.8 \mathrm{wt} \%$ chiral MLC-6428 but without dye using different polarized light at different incident angles, as shown in Figure 4. Figure 4(a), (b) and (c) plot the transmission spectra of the LH CP, RH CP, and LP light, respectively, through a CLC cell without dye at various incident angles. From Figure 4(a), we can see that there is no stop band for the LH CP light. However, due to the substrate surface reflections, the transmittance reaches only $\sim 0.8$ in the visible region at $\theta=0^{\circ}$. For the incident angle $\theta \leq 18^{\circ}$, the sample transmittance decreases only slightly with the increased incident angle. However, for $\theta>18^{\circ}$, the sample transmittance dramatically decreases as the incident angle increases. This explains why the lasing threshold induced by the LH CP light is barely changed if the incident angle is less than $18^{\circ}$, but is dramatically increased when the incident angle is above $18^{\circ}$.

While for the RH CP incident light, there exists a stop band ranging from $550 \mathrm{~nm}$ to $635 \mathrm{~nm}$ at normal incidence, which does not cover the excitation laser wavelength at $\lambda=532 \mathrm{~nm}$. The light loss is mainly due to the surface reflections, same as the LH CP light. As the incident angle increases, besides the increased light loss induced by the substrate surface reflections in the whole visible spectral region, the stop band also produces a noticeable blue shift, as Figure 4(b) shows. When the incident angle is below $18^{\circ}$, although the stop band produces a blue shift, the bandgap does not yet cover the excitation wavelength $(\lambda=532 \mathrm{~nm})$. As the incident angle is above $18^{\circ}$, the short edge of the bandgap starts to cover the pumping wavelength, indicating a portion of the RH CP light of the pump light is reflected by the sample. Further increasing the incident angle causes more RH CP pump light to be reflected because the center of the stop band moves towards $\lambda=532 \mathrm{~nm}$. This explains well our experimental results shown in Figure 3(b): the lasing efficiency is barely changed when the incident angle increases from $0^{\circ}$ to $18^{\circ}$, but is dramatically decreased as the incident angle exceeds $18^{\circ}$. As the incident angle is increased to $\sim 31^{\circ}$, the stop band covers $\lambda=532 \mathrm{~nm}$ due to the blue shift. As a result, most of the $\mathrm{RH}$ circularly polarized pump light is reflected by the sample. As a result, few photons in the $\mathrm{RH}$ circular polarization can enter the sample to excite the dye molecules. Therefore, no lasing 

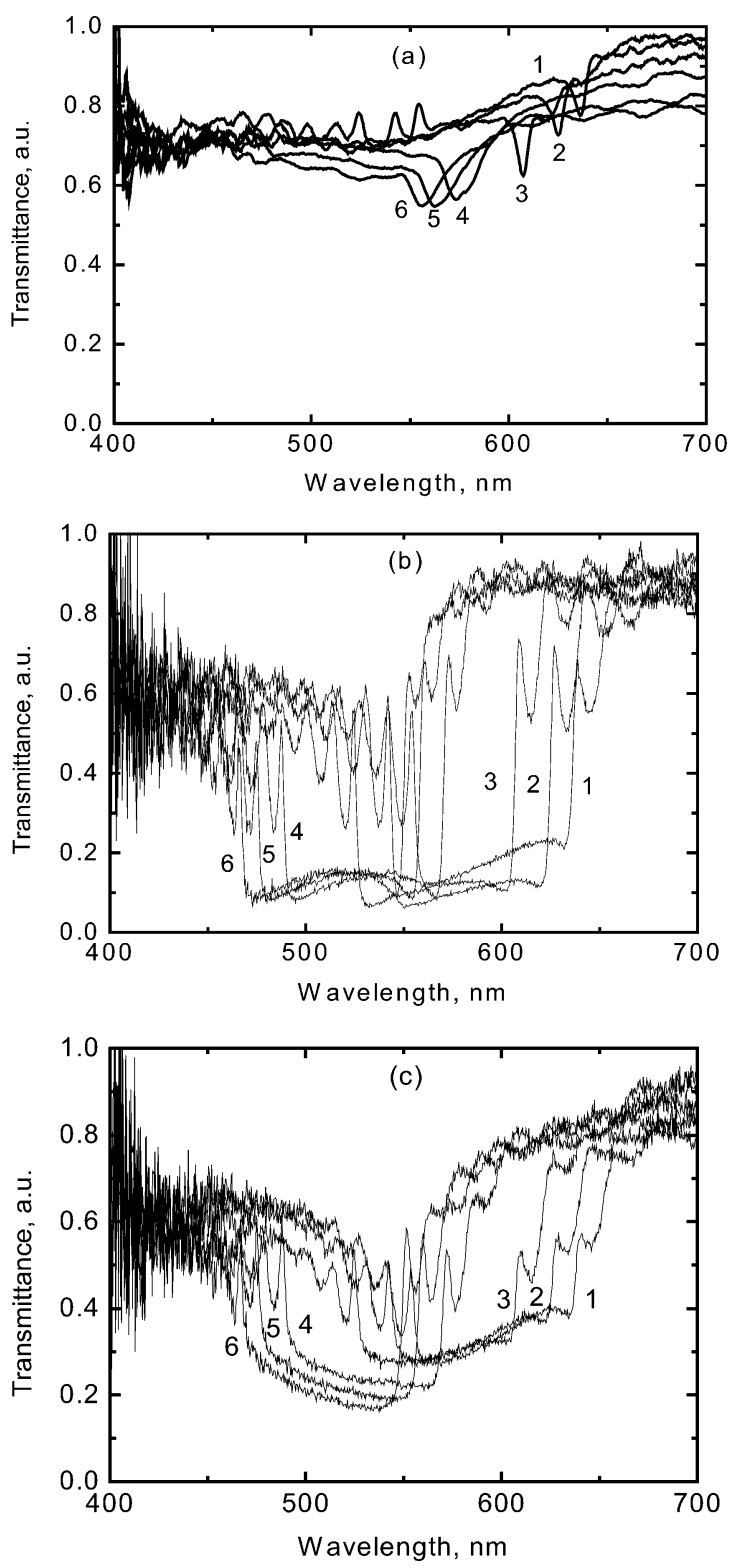

FIGURE 4 Transmission spectra of the CLC without dye for: a) left-handed circularly polarized light, b) right-handed circularly polarized light, and c) linearly polarized light at different incident angles. Curves $1,2,3,4,5$, and 6 are corresponding to the incident angles at $0^{\circ}, 18^{\circ}, 31^{\circ}, 48^{\circ}, 54^{\circ}$, and $60^{\circ}$, respectively. 
can be generated by the $\mathrm{RH} \mathrm{CP}$ pump light for the incident angle above $31^{\circ}$, as shown in Figure 3(c).

A linearly polarized light can be decomposed into a right-handed and a left-handed circularly polarized light. Thus, a LP light also displays a stop band which produces blue shift as the incident angle increases, as shown in Figure 4(c). The laser emission and threshold induced by a LP light also shows the same trend to the incident angle as that by the RH CP light. It is barely changed by the variation of the incident angle when the incident angle is below $18^{\circ}$ and dramatically decreased as the incident angle exceeds $18^{\circ}$. Different from the $\mathrm{RH}$ CP light, the LP light can still generate lasing output from the sample at incident angle above $31^{\circ}$ due to the contribution of the other half $\mathrm{LH}$ CP light. At a large incident angle $\left(>18^{\circ}\right)$, additional optical loss of the RH CP light occurs besides the surface reflections. Thus, the lasing threshold of a LP excitation light is higher than that with LH CP pumping light, as seen in Figure 3.

To study the lasing wavelength effect on the lasing efficiency of the dye-doped CLC, we measured the lasing emission slope from different CLC hosts mixed with $\sim 2 \mathrm{wt} \% \mathrm{DCM}$ and different chiral concentration ranging from $25.7 \mathrm{w} \%$ to $27.7 \mathrm{w} \%$. Results are shown in Figure 5. The emission intensity is significantly enhanced as the excitation energy exceeds the threshold for any lasing wavelengths. However, the threshold excitation energy and lasing efficiency strongly depend

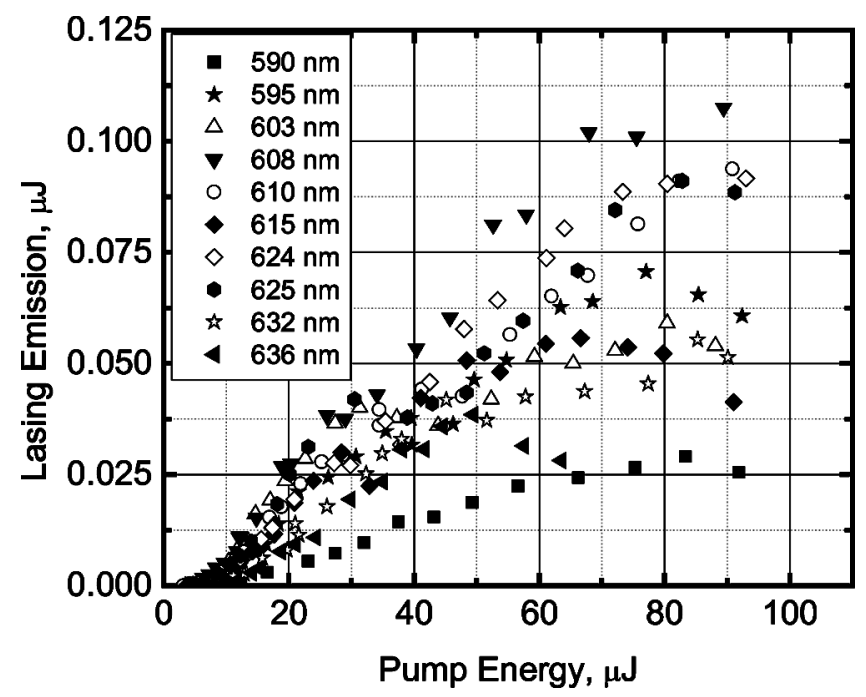

FIGURE 5 Laser emission at different wavelengths. 
on the lasing wavelength, as Figure 5 shows. When the lasing wavelength is at $\lambda=608 \mathrm{~nm}$, the lasing efficiency reaches the maximum and threshold excitation energy is the lowest. If the lasing wavelength is away from $608 \mathrm{~nm}$, higher threshold excitation energy is required and the lasing efficiency is decreased. This behavior stems from the competition between the optical loss and gain. Generally, the lasing emission can be generated through feedback amplification only when the optical gain is lager than the losses in the medium. For the CLC cell, the internal distributed feedback of the CLC photonic band gap provides the amplification of the optical gain traveling in the CLC cell. The laser emission takes place when the amplification of the optical gain traveling in the CLC cell is sufficient to overcome the losses in the medium. A higher optical gain makes the generation of laser emission easier because the optical emission is more effectively obtained by the feedback effect. Typically, the optical gain spectrum has the same spectral appearance as the fluorescence spectrum of the dye. Since the DCM dye exhibits its maximum fluorescence at $\lambda \sim 608 \mathrm{~nm}$, the lowest threshold excitation energy of $\sim 3.14 \mu \mathrm{J} /$ pulse and the highest lasing efficiency occurs for the dye-doped CLC sample when the long edge of the photonic band gap is at $608 \mathrm{~nm}$. When this long edge photonic band gap overlaps with the tail of the fluorescence band, higher threshold energy is required for exciting more optical emission to overcome the loss and finally generating the laser feedback effect. Consequently, the lasing efficiency is decreased.

Since we could obtain the maximum lasing efficiency from the dyedoped CLC mixture with 26.8 wt\% MLC-6248, we chose this mixture to study the effect of the cell gap on the lasing efficiency. The mixture was filled into three different cells with $8 \mu \mathrm{m}, 10 \mu \mathrm{m}$, and $15 \mu \mathrm{m}$ gaps. Figure 6 plots the lasing emission slope for these three samples. The lasing efficiency has the following order: $8 \mu \mathrm{m}<10 \mu \mathrm{m}<15 \mu \mathrm{m}$. This is because the thinner cell has a less distributed feedback layer. As a result, the amplification of the optical gain provided by the distributed feedback in the $8 \mu \mathrm{m}$ cell is less than those in the $10 \mu \mathrm{m}$ and $15 \mu \mathrm{m}$ cells. Therefore, the lasing efficiency obtained from the $8 \mu \mathrm{m}$ cell is lower than those obtained from $10 \mu \mathrm{m}$ and $15 \mu \mathrm{m}$ cells. While for the $10 \mu \mathrm{m}$ and $15 \mu \mathrm{m}$ samples, the situation is complicated. From Figure 6, we can see that at low pumping energy, the lasing efficiency from the $10 \mu \mathrm{m}$ cell is lower than that from the $15 \mu \mathrm{m}$ cell. However, at high pumping energy, the lasing efficiency from the $10 \mu \mathrm{m}$ cell is higher than that from the $15 \mu \mathrm{m}$ cell. It is easy to understand the phenomenon why the lasing efficiency from the $10 \mu \mathrm{m}$ cell is lower than that from the $15 \mu \mathrm{m}$ cell at low pumping energy. Because the $10 \mu \mathrm{m}$ cell has fewer helical layers than the $15 \mu \mathrm{m}$ cell, its optical 


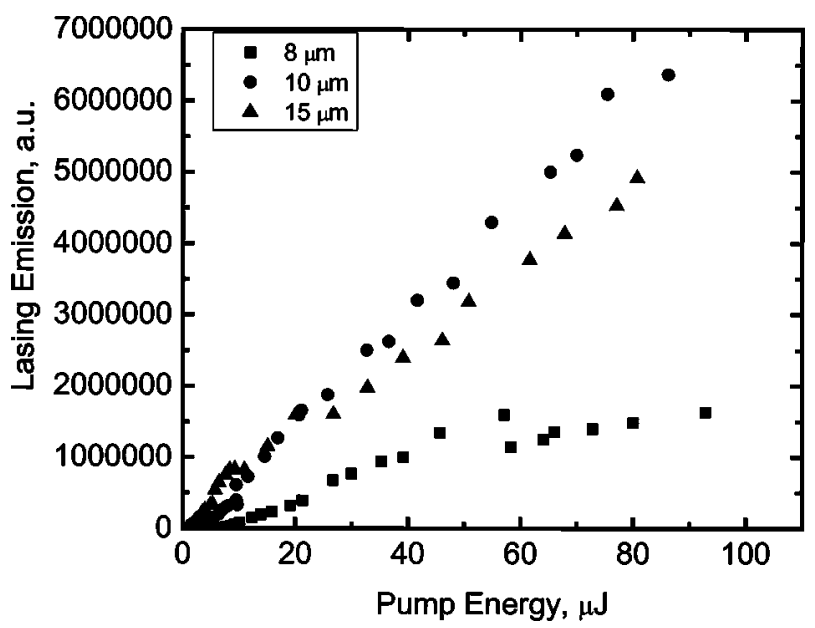

FIGURE 6 Cell gap effect on the lasing emission.

amplification is also less. This explains why the $10 \mu \mathrm{m}$ sample exhibits a weaker lasing emission than the $15 \mu \mathrm{m}$ sample. While at high pumping energy, more photon bleaching and quench effect of the dye molecules occurs in the $15 \mu \mathrm{m}$ sample than in the $10 \mu \mathrm{m}$ one, the lasing efficiency from the $15 \mu \mathrm{m}$ sample is contrarily lower than that from the $10 \mu \mathrm{m}$ sample.

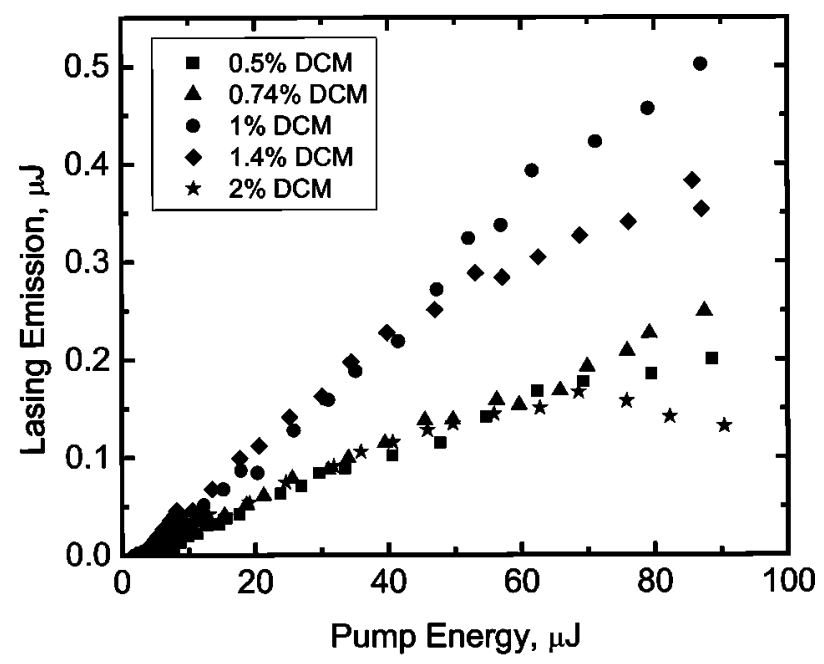

FIGURE 7 Dye concentration effect on the lasing emission. 
To avoid the photon quench in the dye, we investigated the effect of the dye concentration on the lasing efficiency, as shown in Figure 7. In this case, we chose the CLC mixture with $26.8 \mathrm{wt} \%$ MLC-6248 as the CLC host. The DCM dye concentrations studied were: 0.5, 0.75, 1.0, 1.5 , and $2 \mathrm{wt} \%$. These dye-doped CLC mixtures were filled into five $10 \mu \mathrm{m}$ LC cells. As the dye concentration is below $1 \mathrm{wt} \%$, the lasing efficiency increases with the increased dye concentration. However, when the dye concentration is above $1 \mathrm{wt} \%$, the lasing efficiency decreases as the dye concentration increases due to the photon quench effect. The maximum lasing efficiency occurs when the DCM concentration is $1 \mathrm{wt} \%$.

\section{CONCLUSION}

We have investigated the effect of the pumping laser's polarization and incident angles, dye concentration, cell gap and lasing wavelength on the lasing efficiency in dye-doped CLC devices. The dye concentration, cell gap, and lasing wavelength all play important roles on the lasing efficiency. The competing mechanisms include: photon bleaching and quench effect in the dye molecules, competition between the optical gain and optical loss, and optical amplification provided by the distributed feedback which relies on the helical layer number. In this study, we obtained the maximum lasing efficiency $(\sim 0.5 \%)$ at $\lambda \sim 608 \mathrm{~nm}$ from a $10 \mu \mathrm{m}$ CLC sample doped with $1 \mathrm{wt} \%$ DCM when the pump laser beam is left-handed circularly polarized and the incident angle is normal to the sample.

\section{REFERENCES}

[1] Zentgraf, T., Christ, A., Kuhl, J., \& Giessen, H. (2004). Phys. Rev. Lett., 93, 243901.

[2] Wang, L. G., Xu, J. P., \& Zhu, S. Y. (2004). Phys. Rev. E, 70, 66624.

[3] Konorov, S. O., Sidorov-Biryukov, D. A., Zheltikov, A. M., Bugar, I., Chorvat, D., Chorvat, D. Jr., Beloglazov, V. I., Skibina, N. B., Bloemer, M. J., \& Scalora, M. (2004). Appl. Phys. Lett., 85, 3690.

[4] Mok, J. T., Littler, I. C. M., Tsoy, E., \& Eggleton, B. J. (2005). Opt. Lett., 30, 2457.

[5] Andresen, E. R., Thogersen, J., \& Keiding, S. R. (2005). Opt. Lett., 30, 2025.

[6] Wang, Z. X., Lin, C. L., Chow, K. K., Ku, Y. C., \& Bjarklev, A. (2005). Photon. Tech. Lett., 17, 1911.

[7] Mookherjea, S. (2004). Appl. Phys. Lett., 84, 3265.

[8] Qiu, M., Mulot, M., Swillo, M., Anand, S., Jaskorzynska, B., Karlsson, A., Kamp, M., \& Forchel, A. (2003). Appl. Phys. Lett., 83, 5121.

[9] Benabid, F., Knight, J. C., \& Russell, P. St. J. (2002). Opt. Express, 10, 1195.

[10] Wu, S. T. \& Yang, D. K. (2001). Reflective Liquid Crystal Displays, Wiley: New York.

[11] Furumi, S., Yokoyama, S., Otomo, A., \& Mashiko, S. (2003). Appl. Phys. Lett., 82, 16. 
[12] Morris, S. M., Ford, A. D., Pivnenko, M. N., \& Coles, H. J. (2005). J. Appl. Phys., 97, 023103.

[13] Chanishvili, A., Chilaya, G., \& Petriashvili, G. (2003). Appl. Phys. Lett., 83, 5353.

[14] Muñoz, A., Palffy-Muhoray, F. P., \& Taheri, B. (2001). Opt. Lett., 26, 804.

[15] Shirota, K., Sun, H. B., \& Kawata, S. (2004). Appl. Phys. Lett., 84, 1632.

[16] Chanishvili, A., Chilaya, G., \& Petriashvili, G. (2004). Appl. Phys. Lett., 85, 3378. 